health record (EHR) is a powerful tool to capture coded diagnoses at a population level, accurately identifying SLE births is challenging. Our objective was to develop and externally validate algorithms for identifying births to SLE patients.

Methods We used two EHR-based datasets: Vanderbilts Synthetic Derivative and Dukes Clarity. Potential cases had at least 1 SLE code (ICD-9: 710.0 or ICD-10:M32.1*, M32.8, M32.9) and at least 1 ICD-9 or ICD-10 code for pregnancyrelated diagnoses. At Vanderbilt, 100 potential cases were randomly selected for chart review and each classified as a case if SLE was diagnosed by a rheumatologist, nephrologist, or dermatologist. Using this dataset, positive predictive values (PPVs) and sensitivity were calculated for combinations of counts of SLE ICD-9 or ICD-10 codes provided by any clinician and by a rheumatologist (rheumatology coded), antimalarial use, positive ANA, and checked lupus labs (dsDNA, C3 or C4). F-score measured the performance of each algorithm. At Duke, potential cases were compared with the Duke Autoimmunity in Pregnancy Registry; cases outside of this registry underwent chart review. Vanderbilt served as a training set; Duke served as validation.

Results From Vanderbilts 2.8 million subject records, we identified 433 potential cases. Of the 100 cases randomly selected for chart review, 39 had confirmed SLE and a history of a birth. Of Dukes 659 potential cases, 545 were included in a validation set of which 208 had confirmed SLE. In the training set, algorithms with ICD-10 codes had higher PPVs than algorithms with ICD-9 codes (table 1). The algorithm with the highest F-score of $88 \%$ was 4 counts of ICD-9 or ICD-10 codes and checked lupus labs. Algorithms validated well in the Duke dataset. In the validation set, 1 ICD-9 or ICD-10 code (by a rheumatologist) performed best (F-score: 82\%).

Conclusions We have developed and validated algorithms to detect SLE patients with births in the EHR. The highest performing algorithms use SLE ICD-9 or ICD-10 codes and clinical parameters or ICD-10 codes alone. Algorithms using more SLE coded visits have greater PPVs at a cost to sensitivity. While the PPV and sensitivity nears 90\%, EHR cohorts remain complementary to prospective cohorts. However, in the era of big data, developing methods to identify SLE births accurately is critical to examine adverse outcomes such as preterm births.

Funding Source(s): None

Research reported in this abstract was supported by NIH/ NICHD 5K12HD043483-12 (Barnado), NIH/NIAMS 1 K08 AR072757-01 (Barnado), and NIH/NCATSNational 1UL1TR002553 (Duke). The content is solely the responsibility of the authors and does not necessarily represent the official views of the National Institutes of Health.

\section{CONTACT DYNAMICS BETWEEN MESENCHYMAL STEM CELLS AND T CELLS IN LUPUS-PRONE MRL/LPR MOUSE MODEL}

\footnotetext{
${ }^{1}$ Hong Kyung Lee, ${ }^{2}$ Hyung Sook Kim, ${ }^{3}$ Eun Jae Park, ${ }^{4}$ Kyung Sook Kim, ${ }^{5}$ Tae Yong Lee, ${ }^{6}$ Sang-Cheol Bae, ${ }^{7}$ Sang-Bae Han*. ${ }^{1}$ College of Pharmacy, Chungbuk National University; ${ }^{2}$ College of Pharmacy; ${ }^{3}$ College of Pharmacy, Chungbuk National University; ${ }^{4}$ Corestem Ltd; ${ }^{5}$ Corestem Ltd; ${ }^{6}$ Department of Rheumatology, Hanyang University Hospital for Rheumatic Diseases; ${ }^{7}$ College of Pharmacy, Chungbuk National University
}

10.1136/lupus-2019-|sm.52

Background Systemic lupus erythematosus (SLE) is a multiorgan autoimmune disease characterized by autoantibody production and mesenchymal stem cells (MSCs) have emerged as a promising new therapy for the treatment of SLE. MSCs are adult stem cells isolated from various human tissues including bone marrow, adipose tissue, umbilical cord blood, and skeletal muscle; MSCs can differentiate into various cell types and can potentially replace damaged cells in vivo. MSCs suppress $\mathrm{T}$ cell proliferation and cytokine production, reduce $\mathrm{B}$ cell proliferation and antibody secretion, decrease the generation and function of dendritic cells, and reduce the activity of natural killer cells. MSCs also enhance the activity of regulatory $\mathrm{T}$ (Treg) cells. MSCs are thought to inhibit $\mathrm{T}$ cell functions by two different mechanisms: by producing soluble mediators and by direct cellcell contacts. The soluble immunosuppressive factors produced by MSCs include IL10, nitric oxide (NO), tumor growth factor (TGF)-, prostaglandin E2 (PGE2), and indoleamine 2,3-dioxygenase (IDO), all of which can inhibit the functions of major immune cells. Yet, much remains to be learned about the contact-dependent $\mathrm{T}$ cell inhibition by MSCs.

Methods We examined the in vivo efficacy of MSCs in lupusprone MRL/lpr mouse model and examined how MSCs inhibit MRL/lpr $\mathrm{T}$ cells by using time-lapse imaging at the single level.

Results In this study, we show that transfer of human MSCs increased MRL/lpr mouse survival, decreased $\mathrm{T}$ cell infiltration in the kidneys, and reduced $\mathrm{T}$ cell cytokine expression. In vitro, allogeneic mouse MSCs inhibited MRL/lpr T cell proliferation and cytokine production. Time-lapse imaging revealed that MSCs recruited MRL/lpr $\mathrm{T}$ cells establishing long-lasting cellular contacts by enhancing $\mathrm{T}$ cell VCAM-1 expression in a CCL2-dependent manner. In contrast, CCL2 deficient MSCs did not induce T cell migration and VCAM-1 expression, resulting in insufficient cell-cell contact. Consequently, CCL2 deficient MSCs did not inhibit IFN- production by $\mathrm{T}$ cells and upon transfer no longer prolonged survival of $\mathrm{MRL} / \mathrm{lpr}$ mice.

Conclusions Taken together, our imaging study demonstrates that CCL2 enables the prolonged MSC-T cell interactions needed for sufficient suppression of autoreactive $\mathrm{T}$ cells and helps to understand how MSCs ameliorate symptoms in lupusprone MRL/lpr mice.

Funding Source(s): This study was supported by grants funded by the Korean Government (NRF-2017R1A5A2015541 and NRF-2017M3A9B4050336).

\section{HEALTH-RELATED QUALITY OF LIFE IN TUNISIAN PATIENTS WITH SYSTEMIC LUPUS ERYTHEMATOUS}

Amel Rezgui*, Imene Ben Hassine, Monia Karmani, Jihed Anoun, Fatma Ben Fredj, Chedia Laouani. Internal Medicine

10.1136/lupus-2019-Ism.53

Background Systemic lupus erythematosus (SLE) is a chronic inflamatory disease witch can affect different aspects of the patients life, leading to an impairment of health-related quality of life (HRQOL).

The aim of our study was to investigate the role of demographic, clinical, immunological and psychological aspects in influencing the HRQOL of Tunisian patients with SLE and to compare the efficiency of both generic and specific questionnaires of QOL. 
Methods The Medical Outcomes Study Short Form (SF-36) and the Lupus Quality of life (LupusQol) were applied in a cohort of 38 SLE patients. At the time of HRQOL testing, all patients underwent a clinical and laboratory evaluation, together with the measure of disease activity using the Systemic Lupus Erythematous Disease Activity Index (SLEDAI$2 \mathrm{~K})$. In addition, a battery of psychological tests including the Hamilton Anxiety Scale (HAS) and the Hamilton Depression Rating Scale (HAM-D) was applied.

Results The parameters which seemed to greatly influence the impairment of HRQOL were female gender, marital statues, a higher SLEDAI-2k scores as well as higher HAS and HAM-D scores. Arthralgia-arthritis, cutaneous disease activity, neurological disease activity and renal disease activity were correlated negatively with LupusQol subscales. There was a strong positive correlation beteween comparable domains of instruments. Although not as strong as comparable domains, significant correlations were also found between noncomparable domains of LupusQol and PCS and MCS of SF-36.

Conclusions SF-36 and LupusQol were both beneficial instruments in evaluating HRQOL of Tunisian patients with SLE. Anxiety, depression and disease activity in some organs seem to be the major deteminants of HRQOL impairment in SLE patients.

Funding Source(s): None

\section{BLOOD CONCENTRATIONS OF COMPLEMENT SPLIT PRODUCT IC3B AND SERUM C3 ASSOCIATE WITH SYSTEMIC LUPUS ERYTHEMATOSUS DISEASE ACTIVITY}

${ }^{1}$ Alfred H Kim*, ${ }^{2}$ Vibeke Strand, ${ }^{1}$ Deepali P Sen, ${ }^{3}$ Qiang J Fu, ${ }^{4}$ Martin J Schmidt, ${ }^{1}$ John P Atkinson. 'Washington University School of Medicine; ${ }^{2}$ Stanford University School of Medicine; ${ }^{3}$ Saint Louis University College for Public Health and Social Justice; ${ }^{4}$ Kypha, Inc

\subsection{6/lupus-2019-Ism.54}

Background A major unmet need in SLE is the identification of a biomarker that consistently tracks with disease activity. One current approach is measuring complement activation by evaluating consumption of serum C3 and C4. However, since they are acute phase reactants, interpretation of these levels is challenging as serum levels may not decrease until late in a disease flare. $\mathrm{iC} 3 \mathrm{~b}$ is a proteolytically derived molecule of $\mathrm{C} 3 \mathrm{~b}$ and increases with complement activation. $\mathrm{iC} 3 \mathrm{~b} / \mathrm{C} 3$ ratio measures complement consumption relative to production, which may provide for a more accurate assessment of complement activation. We hypothesize that blood iC3b and iC $3 b / C 3$ levels will provide a more specific and reliable marker of complement activation and disease activity in SLE.

Methods 159 consecutive subjects with American College of Rheumatology or Systemic Lupus International Collaborating Clinics classified SLE were enrolled into CASTLE (Complement Activation Signatures in Systemic Lupus Erythematosus), a prospective observational study. Patients with 1-7 study visits were included in this longitudinal analysis. 48 healthy volunteers were enrolled to establish the normal reference iC3b/ C3 ratio. Serum C3 and C4 were measured by nephelometry and blood iC3b levels by a lateral flow assay. SLE disease activity was monitored utilizing the Systemic Lupus Erythematosus Disease Activity Index 2K Responder Index-50 instrument.

Results iC3b/C3 ratio, double-stranded (ds)DNA antibodies (Abs), and supraphysiologic prednisone dose (>7.5 mg/day) each independently correlated with SLE disease activity, employing multilevel multiple logistic regression analysis. Only the $\mathrm{iC} 3 \mathrm{~b} / \mathrm{C} 3$ ratio was significantly associated with clinically meaningful improvements in disease activity among subjects receiving supraphysiologic doses of prednisone. iC3b/C3 outperformed C3 and C4 levels discriminating both active versus inactive SLE disease and major flares versus no disease activity. $\mathrm{iC} 3 / \mathrm{C} 3$, dsDNA Abs, ESR, and supraphysiologic prednisone dose were independently associated with lupus nephritis, while none were associated with SLE rash. The association of $\mathrm{iC} 3 \mathrm{~b} /$ C3 with nephritis was independent of other observed clinical manifestations. Finally, we observed a stronger association of the $\mathrm{iC} 3 \mathrm{~b} / \mathrm{C} 3$ ratio with SLE disease activity in African-Americans compared to Whites.

Conclusions Blood $\mathrm{iC} 3 \mathrm{~b} / \mathrm{C} 3$ correlates with SLE disease activity and clinically meaningful changes. Furthermore, it discriminates between active versus inactive SLE, and major flares compared to those patients without active disease. Differences in the strength of association was observed between races and manifestations.

Funding Source(s): Kypha, Inc. and National Institutes of Health (NIH)/National Institute of Arthritis and Musculoskeletal and Skin Diseases (NIAMS) under Award Number R21AR069833.

\section{POOR SLEEP QUALITY ASSESSED SUBJECTIVELY ASSOCIATED WITH WORSENING SLE DISEASE ACTIVITY}

${ }^{1}$ Alicia M Hinze, ${ }^{2}$ Philip Chu, ${ }^{3}$ Deepali P Sen, ${ }^{3}$ Noor Al-Hammadi, ${ }^{3}$ Yo-El S Ju, ${ }^{3}$ Alfred H Kim*. 'Mayo Clinic College of Medicine; '2Duke University School of Medicine; ${ }^{3}$ Washington University School of Medicine

\subsection{6/lupus-2019-Ism.55}

Background Poor sleep quality is commonly observed in patients with SLE. We hypothesize that poor sleep contributes to worsening SLE. The aims of this study are to evaluate the relationship between subjective sleep measures and SLE activity over time.

Methods A prospective, observational study evaluated the relationship between sleep and SLE disease activity. 151 patients were enrolled. Pittsburgh

Sleep Quality Index (PSQI), Epworth Sleepiness Scale (ESS), Patient Reported Outcomes Measurement Instrument System (PROMIS)-Sleep Related Impairment (SRI), and PROMIS-Sleep Disturbance (SD) survey instruments measured patient reported sleep quality. The population mean for the PROMIS instruments is 50. The SLEDAI-2000 Responder Index-50 (S2K RI-50) was used to define active SLE as S2K RI-50 >4 and worsening SLE at subsequent visits as an increase in S2K RI-50 $\geq 4$. Baseline comparisons were calculated using non-parametric tests. Kaplan-Meier examined the relationship between poor sleep and worsening SLE activity over time.

Results At baseline, the median age was 42, 90.7\% were female, 54.3\% were African American, 24.5\% were on prednisone doses $>7.5 \mathrm{mg} / \mathrm{day}$, and $36.4 \%$ had active SLE. Patients with active SLE had significantly higher SRI scores (median 64.3) vs inactive SLE (median 56.6) as well as significantly higher SD scores (median 58.3 vs 52.2), whereas PSQI and ESS were not significantly different.

Data from 109 patients with $\geq 2$ visits were used for longitudinal studies. Kaplan-Meier analysis, stratified by SRI T-score of $>60$ vs $\leq 60$ demonstrated that worse sleep $($ SRI $>60)$ at the 